\title{
Proposição de uma reserva anatomofuncional, no canal raquidiano, como fator interferente na fisiopatologia das lombal gias e lombociatal gias mecânico-degenerativas
}

\author{
H.A. CECIN \\ Disciplina de Reumatologia da Faculdade de Medicina do Triângulo Mineiro, U beraba, MG.
}

RESUMO - Овј etivo. Testar a hipótese de uma reserva anatomofuncional no canal raquidiano que explique as elevadas prevalências de alterações radiológicas na coluna lombar de indivíduos assintomáticos, as diferentes formas de apresentação clínica das lombalgias e lombociatalgias mecânico-degenerativas e as discrepâncias na sua prevalência.

Casuística e Métodos. Dois grupos de pessoas foram submetidos à tomografia axial computadorizada. Um era de 27 pacientes com Iombalgia e/ ou Iombociatalgia. $O$ outro, de controles pareados, era de 27 indivíduos assintomáticos. Em ambos os grupos, foram medidas as áreas do canal ósseo, do saco dural, a distância interfacetária e a profundidade dos recessos laterais.

Resultados. Os assintomáticos tinham canais raquidianos mais amplos que o de pacientes agudos e crônicos. As variáveis, cujas comparações foram responsáveis por essas diferenças estatisticamente significantes nos crônicos, foram as áreas do canal ósseo em L3-L4, L4-L5, as áreas do

\section{INTRODUÇÃO}

A dor lombar constitui uma grande causa de mor bi dade ${ }^{1-10}$ e incapaci dade ${ }^{2-8}$, sendo sobrepujada apenas pela cefaléia, na escala dos distúrbios dolorosos que afetam o homem ${ }^{2,11}$.

Apesar do progresso da ergonomia aplicada à col una vertebral e do uso de sofisticados métodos de diagnóstico, na última década, as Iombalgias e Iombociatalgias tiveram um crescimento 14 vezes maior que o crescimento da população ${ }^{8}$. O resultado desta situação é grave sob o ponto de vista socioeconômico, causando prejuízos incal culáveis ${ }^{6,9,11-18}$.

No entanto, os conhecimentos existentes sobre o tema não foram suficientes para dissipar a "densa névoa" de desinformação que envolve alguns de seus aspectos $2,10,19,20$.

Ao longo da nossa prática médica, a constatação de alterações radiológicas na coluna lombar, em pessoas assintomáticas - e que, posteriormente, saco dural em L4-L5, L5-S1 e a profundidade dos recessos laterais em L 4-L5; e nos agudos: a área do canal ósseo em L3-L 4, L4-L 5, L5-S1, área do saco dural em L4-L5, L5-S1, o diâmetro sagital em L5S1 e a profundidade do recesso lateral direito em L4-L5.

ConClusões. As alterações radiológicas e ausência de sintomas no grupo-controle foram atribuídas a uma maior reserva anatomofuncional no canal raquidiano; a existência de casos agudos e crônicos, a uma reserva menor. Esta reserva, maior ou menor, seria um "fator segurança" que se distribuiria diferencialmente em assintomáti cos, agudos e crônicos, e seria o el emento capaz de influenciar a prevalência, a presença ou ausência de dor lombar em indivíduos com alterações radiológicas.

UNITERMOS: Reserva anatomofuncional. Dor lombar. Lombociatalgias mecânico-degenerativas. Canal raquidiano. Alterações radiológicas em assintomáticos.

foi confirmada por estudos independentes aqui ${ }^{21-23}$ e al hures, 24-38 - fez surgir, em nós, al gumas intrigantes interrogações:

1) Qual a razão dessa ascendente prevalência, constatada por diversos estudos epidemiológi$\cos ^{2,4,8,15,18,39-46}$ ?

2) Os estudos acima mencionados, ao apontarem uma prevalência de lombal gias e lombociatalgias da ordem de $55 \%$ a $65 \%$, em diferentes grupos populacionai ${ }^{3,4}$, levam à indagação: então, por que os restantes $45 \%$ e $35 \%$ não apresentam sintomas, se as suas colunas lombares foram submetidas a um semel hante estresse físico e estando em semeIhantes condições de trabalho?

3) Quais as razões de algumas pessoas, desse mesmo contingente, serem refratárias à dor, não obstante a sua coluna lombar apresente, em mai or ou menor grau, intensas alterações radiológicas?

Se essas indagações for em respondidas - éo que tentaremos fazer —, a complexa e difícil fisiopato- 
logia ${ }^{5,10,20,47}$ da dor lombar, tal vez, receba uma contribuição que diminua essas dificuldades.

As causas mais freqüentes das lombalgias e lombociatal gias, acima referidas, são de natureza mecânico-degenerativa. Sua produção tem origem em desordens estruturais, desvios biomecânicos, ou na interação desses dois fatores. Existe consenso quanto à partici pação de um processo degenerativo estrutural (primitivo ou secundário ao desvio biomecânico) na deflagração das principais causas, abaixo mencionadas, da síndrome dolorosa lombar 1,7,9,12,18,48-56: discartroses, protrusões discais focais, protrusões discais difusas, osteoartrose zigoapofisária, espondilolisteses, hérnias discais, degeneração do ligamento amarelo, escolioses e hiperlordoses.

Considerando que as alterações radiológicas, tanto em indivíduos assintomáticos como em portadores de lombal gi as e ciatal gias, podem ser devidas a essas mesmas causas, é intrigante o fato de nos primeiros não existir produção de sintomas, enquanto que nos últimos, sim.

A resposta para essas indagações pode estar na fisiopatologia da dor lombar, principalmente naquel es aspectos concernentes às relações anormais entre os elementos nervosos e as estruturas osteoarticulares e discais adjacentes. Como essas relações ocorrem dentro de um espaço real, é de se antever que tanto maior será o conflito entre estas estruturas (com produção de dor) quanto mais exíguo ele for.

Assim sendo, a exata compreensão do fenômeno doloroso lombar e de sua fisiopatologia justifica uma revisão dos conhecimentos anatômicos, biomecânicos e fisiol ógi cos sobre a coluna lombar; e é bastante provável que, por esse caminho, passem as respostas que esclareçam a intrigante indagação levantada e, por conseguinte, nos possibilite alcançar o objetivo adiante exposto.

A coluna lombar tem, como base anatômica e funcional, a tríade articular formada por uma juntura fibroelástica intervertebral — o disco-, duas junturas sinoviais e um corpo vertebral ${ }^{57}$. Esse conjunto de três compartimentos é estabilizado por um aparel ho ligamentar que permiteà raque movimentos pela ação de uma complexa coordenação entre função muscular e gravidade ${ }^{57,58}$.

O disco intervertebral é uma estrutura anatômica que tem função de absorver choques e permitir um certo grau de movimento à col una vertebral. Quanto maior for o seu conteúdo em água, maior é a sua capacidade de absorção de choques ${ }^{59}$.

Sua eficiência biomecânica diminui com o aumento da idade devido à sua desidratação, quando também ocorre aumento do estresse mecânico. A integridade desse verdadeiro amortecedor de choques é então afetada ${ }^{9,60}$.

Por outro lado, a el evada concentração de íons hidrogênio decorrente do metabolismo anaeróbico do disco - que, por ser uma estrutura avascular, se nutre por difusão passiva - pode causar dor, via receptores químicos na zona fronteiriça entre o núcl eo e o anel fi broso, local das primeiras fissuras. Esse fato éo primeiro sinal de degener ação discal ${ }^{61}$.

As modificações da estrutura do disco, a sua dessecação e a conseqüente diminuição da altura do espaço intervertebral, por ele ocupado, afetam o equilíbrio mecânico existente com as articulações zigoapofisárias e os ligamentos, pois há uma transferência de carga do primeiro para os outros dois.

Aos $20 \%$ da carga vertical, aplicada normalmente sobre o pedículo e facetas articulares, somam-se outros $20 \%$ ou mais. Os resultados dessa sobrecarga se manifestam por: degeneração, desalinhamento das facetas articulares ${ }^{62,63}$, distensão e afrouxamento da cápsula articular, instabilidadee subluxação subseqüentes.

O disco degenerado avança alguns milímetros para trás, e essa mobilidade cria um estresse anormal no conjunto, gerando um ciclo vicioso de forças excessi vas e anormais sobre a tríade articular. As conseqüências finais desse fenômeno são modificações na geometria espacial da col una lombar, com reflexos sobre o vol ume do canal raquídeo e sobre as estruturas nervosas e os seus vasa nervorum $^{2,4,35,57,58,64-68}$. Em síntese, os conhecimentos atuais de Anatomia, Biomecânica e Fisiopatologia colocam em destaque o papel dos fatores mecânicos, degenerativos e bioquími cos que, ao interagirem dentro de um espaço em movimento, produzem al terações histológicas e radiológicas.

A ilação prática, oriunda destes conhecimentos anatomofisiológicos, coloca em relevo o papel do fator mecânico e bioquímico na fisiopatologia da dor lombar e, a partir dela, se vislumbra uma explicação para as questões levantadas. Ora, ocorrendo esse fenômeno fisiopatológico em determinadas pessoas, por qual motivo não se traduziria por dor lombar em umas, enquanto que em outras, sim?

E nfim, e redargüindo: se alterações radiol ógicas ocorrem como conseqüência de modificações estruturais e mecânicas, por que motivo o conflito resultante, em algumas pessoas, produz sintomas, enquanto que em outras, não? Existiria, nessas e naquelas, algum fator que se distribuiria diferencialmente, facilitando ou dificultando o aparecimento da dor lombar?

Em face do exposto, as dúvidas levantadas ao longo dessa introdução, e as respostas esperadas, 
tornam oportunas quaisquer tentativas visando superar as dificuldades, ainda existentes, no entendimento da fisiopatologia da dor lombar.

\section{OBJ ETIVOS}

Comparar, por meio de medi das realizadas pela tomografia axial computadorizada, os parâmetros vertebrais implicados na produção de lombal gias e Iombociatal gias (área do canal ósseo, área do saco dural, diâmetro sagital, distância interfacetária, profundidade dos recessos laterais) de um grupo de indivíduos assintomáticos e, de outro, de pessoas com lombal gia e lombociatal gia de etiologia mecânico-degenerativa, para testar a hipótese da possível existência de uma reserva anatomofuncional no canal raquidiano (fig. 1) que possa explicar:

- discrepâncias na preval ência de lombalgias e lombociatal gias, em indivíduos submetidos a semel hantes cargas e em iguais condições de trabaIho, conforme estudos epidemiológicos realizados no Brasil e no exterior;

- a ausência de sintomas em portadores de alterações radiológicas vertebrais na coluna lombar, demonstradas por qualquer dos métodos de uso corrente no nosso meio;

- a existência de quadros agudos e quadros crônicos, clinicamente diferentes entre si;

- e, em existindo a referida reserva anatomofuncional: rediscutir o papel da propedêutica clínica no diagnóstico das I ombal gias el ombociatal gias, atualmente relegado a um segundo plano, diante do impacto provocado por novos e sofisticados métodos de diagnóstico.

\section{CASUÍSTICA E MÉTODOS}

\section{Casuística}

A amostra é formada por 54 pessoas, distribuídas em casos (pacientes) e controles (assintomáticos).

\section{Seleção de casos e controles}

\section{Casos}

O grupo de pacientes, doravante chamado de sintomático, é constituído de 27 casos de pacientes com lombal gia, Iombociatal gia e/ou ciática de causa mecânico-degenerativa, sendo 15 homens e 12 mulheres.

Como lombalgia, foram consideradas todas as condições de dor, dolorimento, rigidez, ou fadiga, localizadas na região inferior do dorso, em uma área situada entre o último arco costal e a prega glútea. Lombociatalgia foi definida como sendo a

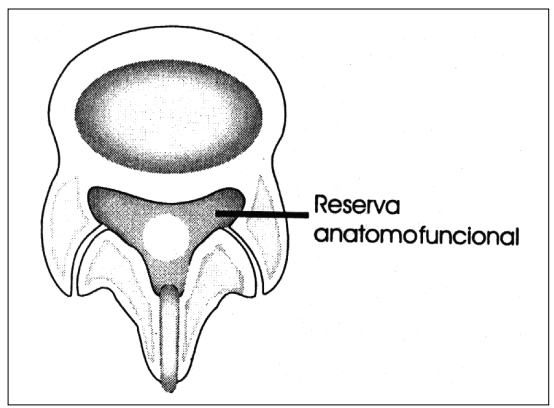

Fig. 1 - Espaço epidural (reserva anatomofuncional ). dor que se irradiava da região do dorso, acima delimitada, para uma ou ambas as pernas. Consideramos como ciática esta situação acima referida, quando, além do fenômeno doloroso, os pacientes apresentavam déficit motor e neurossensorial 3,17,69.

Como causas mecânico-degenerativas, foram caracterizadas aquelas Iombalgias, Iombociatalgias e/ou ciáticas, nas quais foi possível excluir uma etiologia inflamatória, infecciosa, metabólica ou tumoral ${ }^{52,65,70,71}$ para cuja produção concor rem as seguintes entidades ${ }^{6}$ : as protrusões discais; as discartroses ou espondiloses; as hérnias discais; a degeneração do ligamento amarel o; as osteoartroses zigoapofisárias; as espondilolisteses; e estreitamento do canal raquidiano.

Para apurar possíveis diferenças, nas formas clínicas de apresentação das Iombal gias e lombociatal gias, os paci entes do grupo sintomático foram separados em agudos e crônicos.

Critérios de distinção entre pacientes agudos e crônicos (segundo experiência pessoal)

Dos 27 pacientes, 17 eram casos crônicos e dez, agudos.

Foram considerados agudos, ou em fase de agudização, aqueles pacientes que, durante o exame, apresentavam um ou mais dos sintomas e sinais abaixo relacionados: 1 ) dor lombar e/ou ciática e/ou ciatalgia intensa; 2) extrema dificuldade para deambular, com necessidade de auxílio para a marcha (órtese ou apoi o em outra pessoa); 3) agravamento e exacerbação da dor pel os movi mentos de flexão, extensão e inclinaçãolateral ; 4) presença de sinal de Làsegue positivo entre $0^{\circ}$ e $70^{\circ}$ e/ou sinal Bragard; 5) presença da manobra de Valsal va positiva; 6) diminuição da força muscular do hálux contra uma resistência oposta pelo examinador; 7) sinal "das pontas" (de De Sèze) positivo; 8) abolição do reflexo patelar ou aquileu.

F oi estabel ecido, em 30 dias, o tempo máximo de presença de um ou mais desses sinais e sintomas.

Como crônicos, foram considerados aqueles pacientes que, além da dor lombar, ciatal gia ou ciática, denotavam um ou mais dos seguintes sinais e sinto- 
mas: 1) claudicação intermitente dos membros inferiores; 2 ) deambulação possível, sem necessi dade de auxílios para marcha; 3) dor à extensão da coluna lombar; 4) dor nas panturrilhas; 5) sinal de Làsegue negativo; 6) ciatal gia bilateral;

O tempo de duração dos sintomas, para caracterizar a cronicidade, foi estabel eci do em mais de 30 dias.

\section{Controles}

O grupo controle de 27 pessoas, que de agora em diante será chamado de assintomático, foi formado de 16 homens e 11 mulheres. $\mathrm{Na}$ composição desse grupo, com o objetivo de, pel o menos parcialmente, atenuar a ação de variáveis modificadoras e/ou tendenciosidades devido ao caráter da casuística, optamos pelo estudo pareado por idade, sexo e peso. Desta forma, para cada paciente, sempre que possível, selecionamos uma pessoa sem dor lombar, do mesmo sexo e de i dade e peso similares (com variação máxima de dois anos para mais ou para menos, e de $2 \mathrm{~kg}$ para mais ou menos, respectivamente). A certeza de que os componentes do grupo controle eram realmente assintomáticos foi obtida depois de uma rigorosa investigação que incluía entrevista com anamnese e exame físico.

Foram excluídas desse grupo as pessoas com história de acidente automobilístico, que fizeram radiografias da coluna lombar (por qualquer motivo), ou que tinham déficit de memória.

\section{Metodologia (programas, testes estatísticos e re- cursos)}

\section{Programas e instrumentos}

A análise estatística foi feita em um microcomputador AT-386, utilizando-se os seguintes programas: E pinfo, versão 1991 (março) do Center for Disease's Control, E pidemiology Program Office, de Atlanta, Georgia (EUA), e da World Health Organization (Global Program of AIDS), Genebra, Suíça; Microstat...E cosoft I nc. 1984; Minitab, Inc., versão 82.1.

\section{Testes estatísticos}

As igualdades das variâncias foram testadas pela estatística de Bartlett ${ }^{72,73}$, tendo sido detectada heterocedasticidade para os parâmetros vertebrais e homocedasticidade para as variáveis idade, peso e altura.

Em seguida, a comparação entre os grupos, no que se refere às variáveis heterocedásticas, foi feita pelo teste de Kruskall-Wallis (simultaneamente, para os assintomáticos, agudos e crônicos). Quando houve diferenças estatisticamente significantes ou quando os níveis de significância indicas-

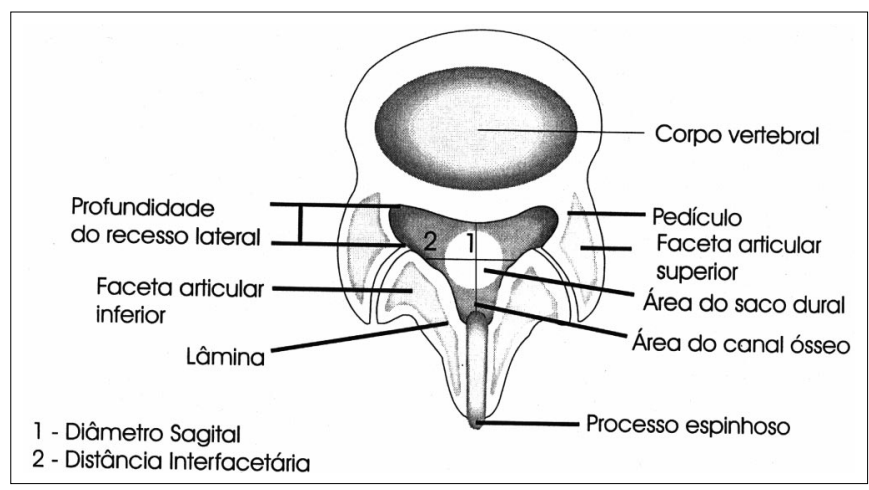

Fig. 2 - Parâmetros vertebrais.

sem uma forte tendência nessa direção, foi realizado o teste de Willcoxon-Mann-Whitney, para verificar as diferenças específicas. Para a idade, peso e altura, testou-se a simetria das distribuições, mediante comparação das médias e medianas entresi.

Garantidas a simetria e a homocedasticidade das distribuições, os grupos foram comparados, segundo essas variáveis, pela análise de variância; quando se constatou alguma diferença, usou-se o método da "mínima diferença significante", para comparação entre pares de grupos ${ }^{74}$.

O teste do $\chi^{2}$ (qui-quadrado) e o teste exato de Fischer foram usados para comparar os grupos, segundo variáveis qualitativas, conforme a indicação e segundo os valores esperados.

Estabelecemos em 5\% o nível de significância estatística.

\section{ESTUDO RADIOLÓGICO}

\section{Aparel hos}

Os aparel hos usados para o estudo tomográfico computadorizado foram da marca GE 8.800 e um Philips 640; em apenas um controle foi usado um aparel ho Toshiba.

As radiografias convencionais foram feitas com um aparelho Siemens de 500mA e um aparel ho Fotorax 300mA.

\section{Técnica do exame tomográfico}

Como primeira etapa, efetuou-se um scout-view (visão panorâmica da coluna lombar) lateral em todos os examinados, para estabelecer os níveis de corte, que se iniciavam na metade do corpo da $3^{3}$ vértebra lombar e terminavam na plataforma da primeira vértebra sacra.

A escolha desses três últimos segmentos para estudo deveu-se à maior prevalência de lesões nesses níveis ${ }^{26,28,75}$.

Os parâmetros avaliados foram os seguintes: área do canal ósseo; área do saco dural; diâmetro 
Fig. 3 - Área do canal ósseo.
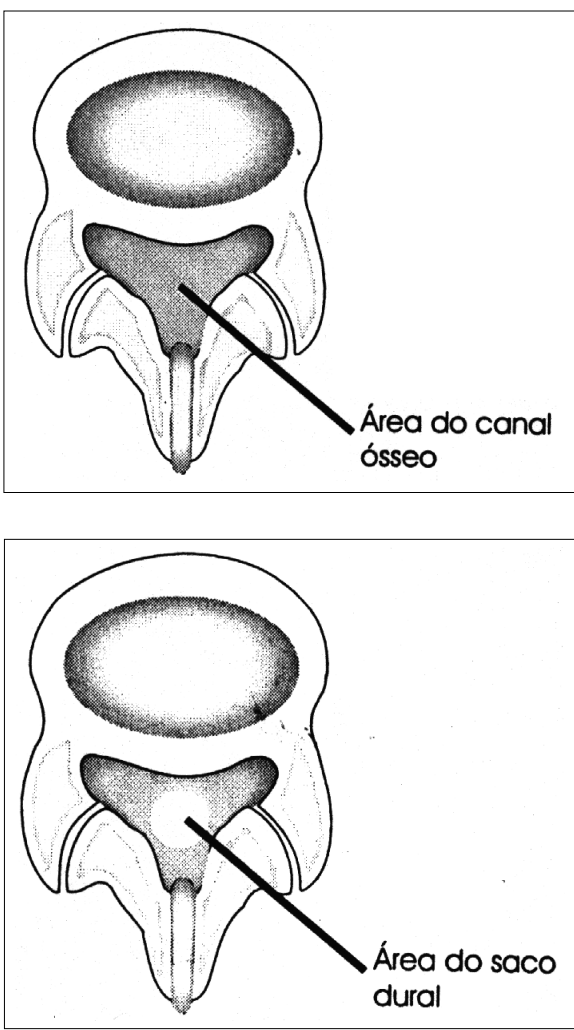

Fig. 4 - Área do saco dural.

sagital; distância interfacetária; profundidade dos recessos laterais (fig. 2).

A área do canal ósseo - medida na porção média do corpo vertebral (no corte que passava, exatamente, no meio do corpo vertebral) (fig. 3).

A área do saco dural - mensurada naqueles cortes que tangenciavam o espaço discal (fig. 4).

O diâmetro sagital - medido também no meio do corpo vertebral, de um ponto o mais posterior do corpo vertebral, até outro, o mais ventral do processo espinhoso correspondente (fig. 5).

A distância interfacetária - avaliada entre dois pontos, os mais mediais, das facetas das articulações zigoapofisárias (fig. 6).

As profundidades dos recessos laterais - medidas na porção superior do pedículo (fig.7).

As medições foram processadas por meios eletrônicos. A margem de erro nestes processos é da ordem de $0,01 \%$.

Técni ca do exame radiológi co convencional

As radiografias convencionais foram executadas nas incidências de frente e perfil, usando-se $200 \mathrm{~mA} / \mathrm{s}$, com pequena variação de quilovol tagem, dependendo da espessura da pessoa radiografada.

\section{RESULTADOS}

A profissão dos componentes dos dois grupos era

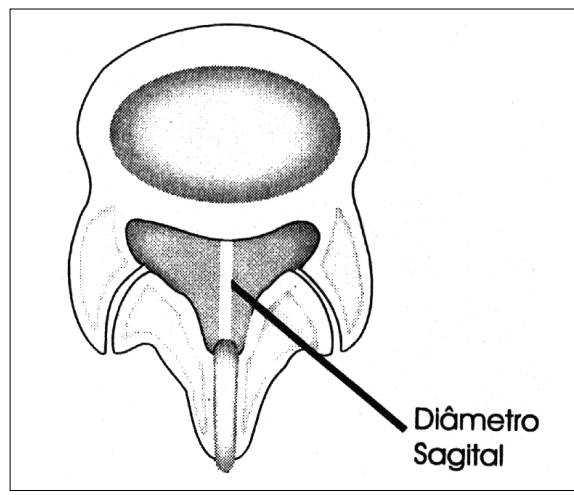

Fig. 5 Diâmetro sagital.

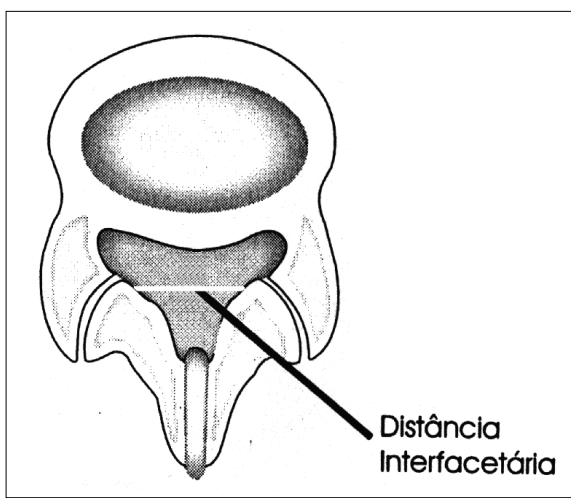

Fig. 6 Distância interfacetária.

formada por pessoas de diferentes grupos ocupacionais. Todos os assintomáticos eram brasileiros. Dos sintomáticos, 25 eram brasileiros; um, português e um, holandês. Quanto à cor da pele dos assintomáticos, um era negro, um, mulato, e 25, brancos. Os sintomáticos eram todos brancos.

Os sintomas e sinais dos pacientes, peculiares a agudos e crônicos, encontram-se nos gráficos 1, 2 e 3.

Nos gráficos 4 e 5, são vistas as al terações tomográficas em assintomáticos e sintomáticos.

Para verificar a homogeneidade das amostras, segundo os critérios de pareamento, os casos e controles foram distribuídos segundo as variáveis idade, sexo, peso, estatura, e comparados entre si. Por não ter havido diferenças estatisticamente significantes $(p>0,05)$, o pareamento foi considerado eficiente.

Os assintomáticos e sintomáticos, esses agora separados em agudos e crônicos, foram comparados simultaneamente entre si, para verificar possíveis diferenças quanto a idade, peso e estatura.

As igual dades das variâncias, testando simultaneamente essas variáveis em assintomáticos, agudos e crônicos, indicaram homocedasticidade, segundo a estatística de Bartllet. Portanto, também nesse aspecto, a amostra é homogênea. $E \mathrm{~m}$ seguida, os assintomáticos, agudos e crônicos foram distribuídos segundo as variáveis de estudo: área do canal ósseo, área do saco dural, distância inter- 


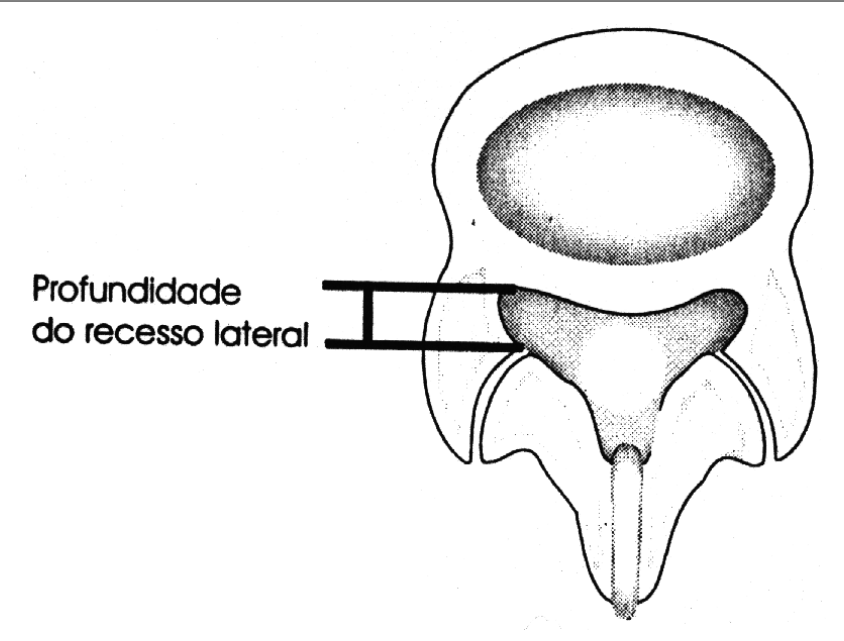

Fig. 7 - Profundidade do recesso lateral.

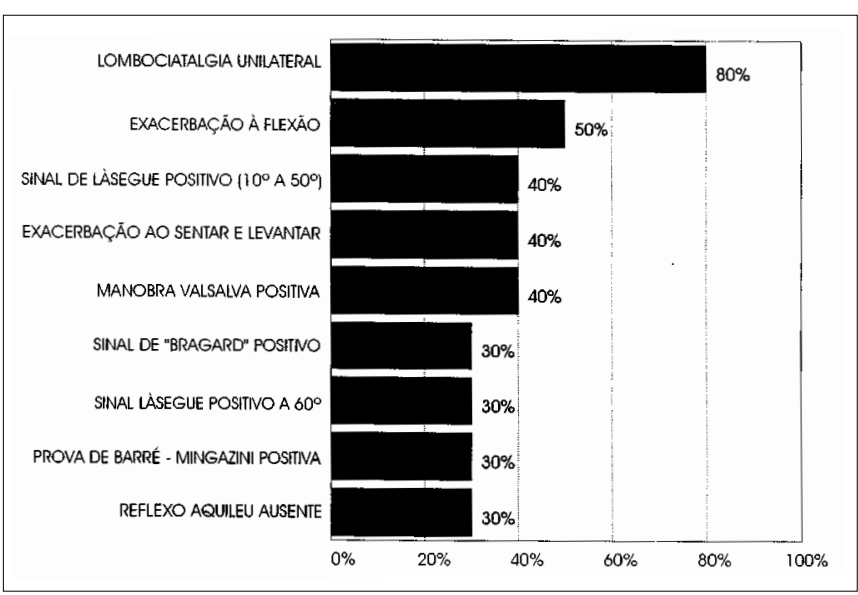

Gráfico 1 - Achados clínicos nos sintomáticos agudos $(n=10)$.

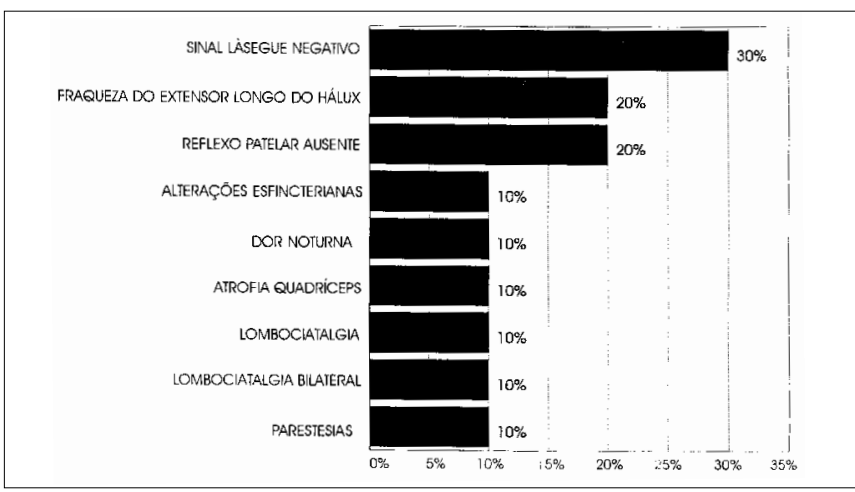

Gráfico 2 - Achados clínicos nos sintomáticos $(n=10)$.

facetária, diâmetro sagital e profundidade dos recessos laterais.

A distribui ção segundo a média, mediana e desvio-padrão mostrou que os assi ntomáti cos tiveram, com duas raras exceções, médias e medianas siste mati camente superi ores às dos agudos e crôni cos. A igualdade de variâncias, mediante comparação si -

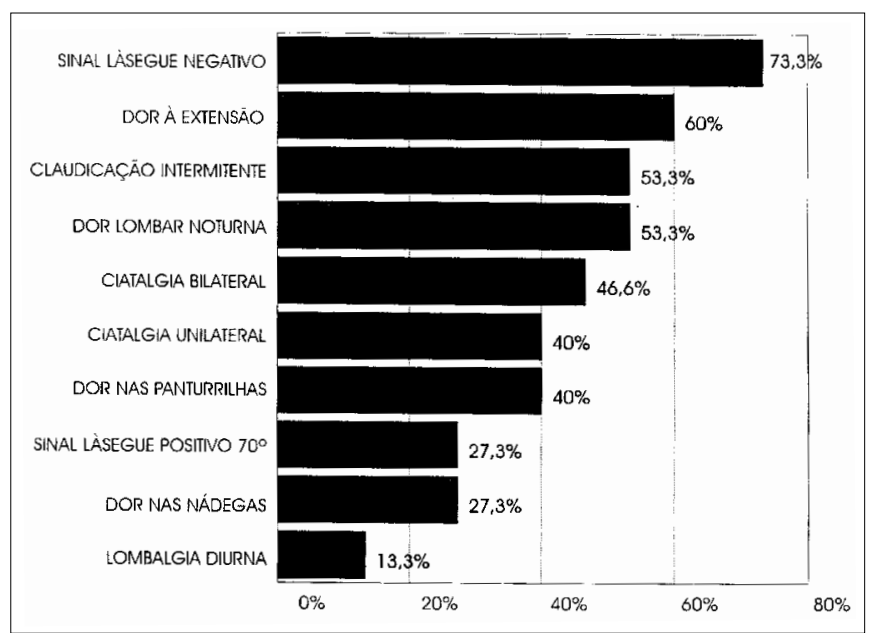

Gráfico 3 - Achados clínicos nos sintomáticos crôni cos $(n=17)$.

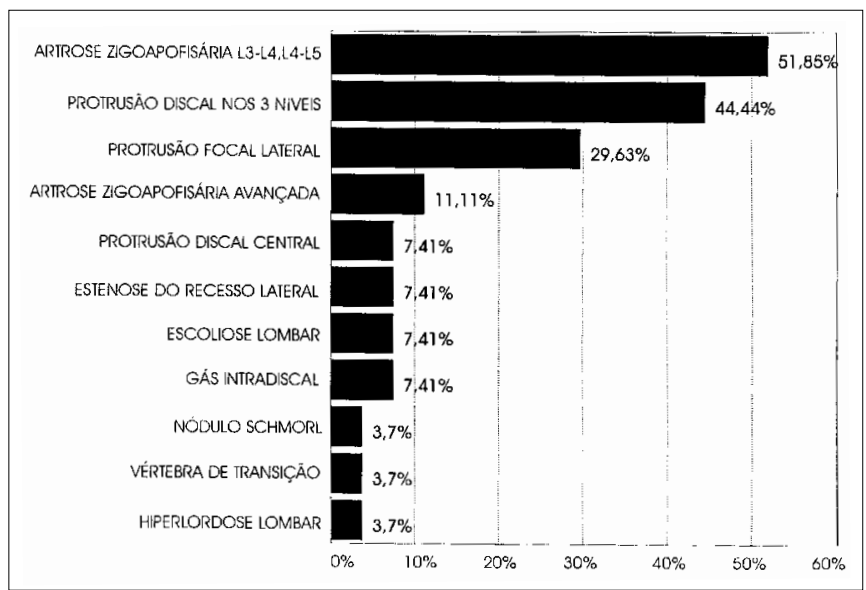

Gráfico 4 - Achados tomográfi cos $(81,5 \%)$ nos assintomáticos $(n=27)$.

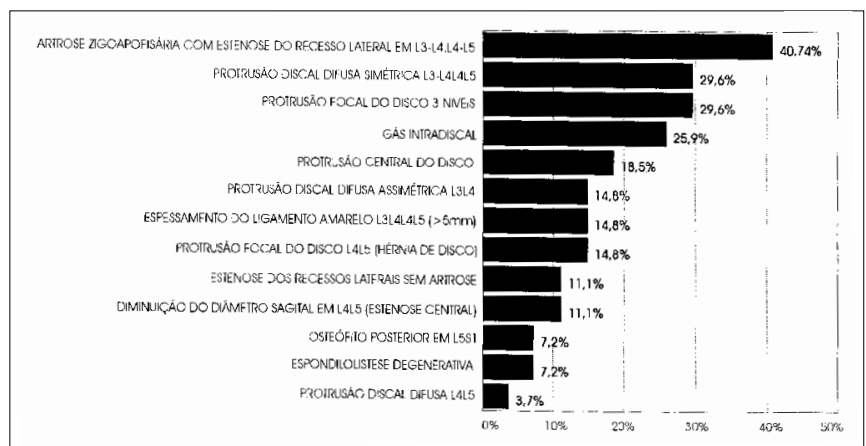

Gráfico 5 - Achados tomográficos $(92,6 \%)$ nos sintomáticos $(n=27)$.

multânea dos três grupos pela estatística de Bartllet, indicou a ocorrência de heterocedasticidade entre todas as variáveis analisadas.

Para verificar diferenças entre os casos e controles segundo a presença ou não de alterações tomográficas, uns e outros foram comparados entre si, conforme se pode ver na tabela 1 . 


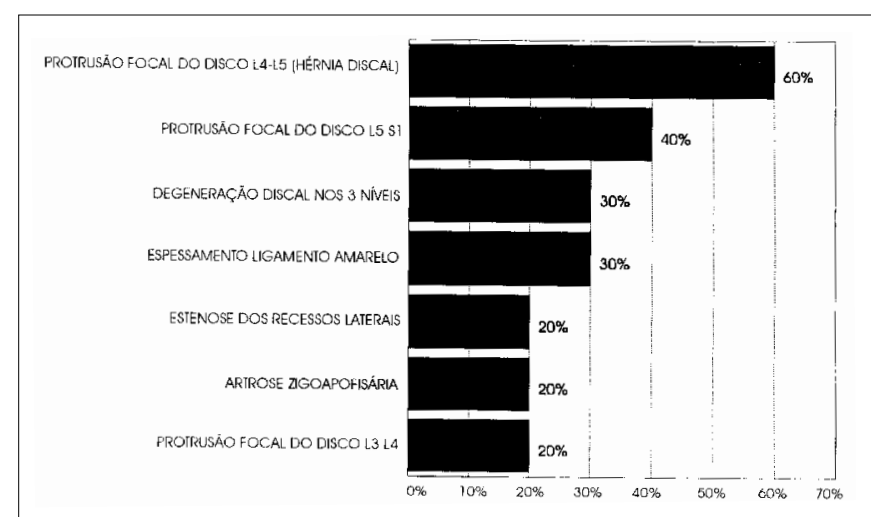

Gráfico 6 - Achados tomográficos nos sintomáticos agudos $(n=10)$.

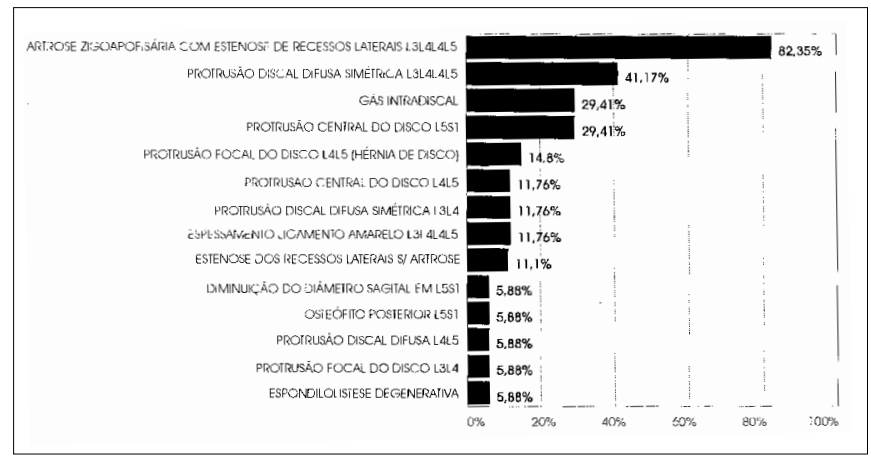

Gráfico 7 - Achados tomográficos nos sintomáticos crônicos $(n=17)$.

Essa tabela indica que não houve diferenças estatisticamente significantes, quando se comparou a prevalência de alterações tomográficas nos assintomáti cos e sintomáticos. No que tange a esse aspecto, são, portanto, semel hantes os percentuais de prevalência $(81,5 \%$ × 92,6\%).

Por outro lado, conforme pode ser notado nos gráficos 4 e 5, a artrose zi goapofisária, a protrusão discal difusa e as protrusões focais foram os achados mais freqüentes, tanto em assintomáticos como em sintomáticos. A se consi derar apenas esses últimos achados, verifica-se serem também semel hantes os percentuais de prevalência, em relação às causas geradoras dessas alter ações mencionadas.

Mesmo quando os sintomáticos são desdobrados em agudos e crôni cos (gráficos 6 e 7), continuou não havendo diferenças estatisticamente significantes quanto às alterações tomográficas, quando eles foram separadamente comparados com os assintomáticos, como mostram as tabelas 2 e 3.

Também no que concerne ao raio- $X$ convenci onal, assintomáticos e sintomáticos não apresentaram diferenças estatisticamente significantes $(p>0,05)$, quando comparados entre si.

$\mathrm{Na}$ tabela 4, estão sumariadas as várias comparações realizadas entre assintomáticos, agudos e
Tabela 1 - Distribuição dos casos e controles, segundo as prevalências de alterações tomográficas

\begin{tabular}{|lcc|}
\hline & \multicolumn{2}{c|}{ Alterações } \\
\cline { 2 - 3 } & Sim & Nãomográficas \\
Casos (sintomáticos) & $25(92,6 \%)$ & $2(7,4 \%)$ \\
Controles (assintomáticos) & $22(81,5 \%)$ & $5(18,5 \%)$ \\
\hline \multicolumn{2}{l}{ Teste exato de Fischer; $\mathrm{p}=0,4202}$. \\
\hline
\end{tabular}

Tabela 2 - Distribuição dos assintomáticos e sintomáticos agudos, segundo a prevalência de alterações tomográficas

\begin{tabular}{|lll|} 
& \multicolumn{2}{c|}{ Alterações tomográficas } \\
\cline { 2 - 3 } & \multicolumn{1}{c|}{ Sim } & Não \\
Assintomáticos & $22(81,5 \%)$ & $5(18,5 \%)$ \\
Sintomáticos agudos & $10(100 \%)$ & $0(0 \%)$ \\
\hline Teste exato de Fischer; $\mathrm{p}=0,295$. & & \\
\hline
\end{tabular}

Tabela 3 - Distribuição dos assintomáticos e sintomáticos crônicos, segundo a prevalência de alterações tomográficas

\begin{tabular}{|lll|} 
& \multicolumn{2}{c|}{ Alterações tomográficas } \\
\cline { 2 - 3 } & \multicolumn{1}{c|}{ Sim } & Não \\
Assintomáticos & $22(81,5 \%)$ & $5(18,5 \%)$ \\
Sintomáticos crônicos & $25(96,3 \%)$ & $2(3,7 \%)$ \\
\hline Teste exato de Fischer; $\mathrm{p}=0,42$. & & \\
\hline
\end{tabular}

crônicos. Em razão da heterocedasticidade detectada, a comparação foi feita pelo teste de KruskallWallis (para os parâmetros vertebrais) e pela ANOVA (análise de variância) para idade, peso e altura.

$\mathrm{Na}$ tabela 4, podemos observar que a área do canal ósseo em L 3-L 4 e L4-L5, a área do saco dural em L4-L5, o di âmetro sagital L5-S1 e a idade mostraram diferenças estatisticamente significantes, quando suas médias foram comparadas simultaneamente entre si. E m relação à área do saco dural em L 3-L 4 e L 5-S1 e às profundidades dos recessos laterais em L4-L5, verificou-se uma forte tendência nessa di reção, uma vez que as diferenças encontradas envolvem números da ordem de déci mos de milímetros.

Para verificar diferenças específicas entre assintomáticos e agudos, assintomáticos e crônicos, e entre agudos e crônicos, as variáveis que mostraram diferenças estatisticamente significantes entre si (quando se compararam, simultaneamente, os três grupos pelo teste de Kruskall-Wallis e a ANOVA), foram testadas duas a duas pelo teste de Willcoxon-Mann-Whitney (parâmetros vertebrais) e pelo teste da "mínima diferença significante" (idade, peso e altura). Essas comparações são vistas nas tabelas 5, 6 e 7. 


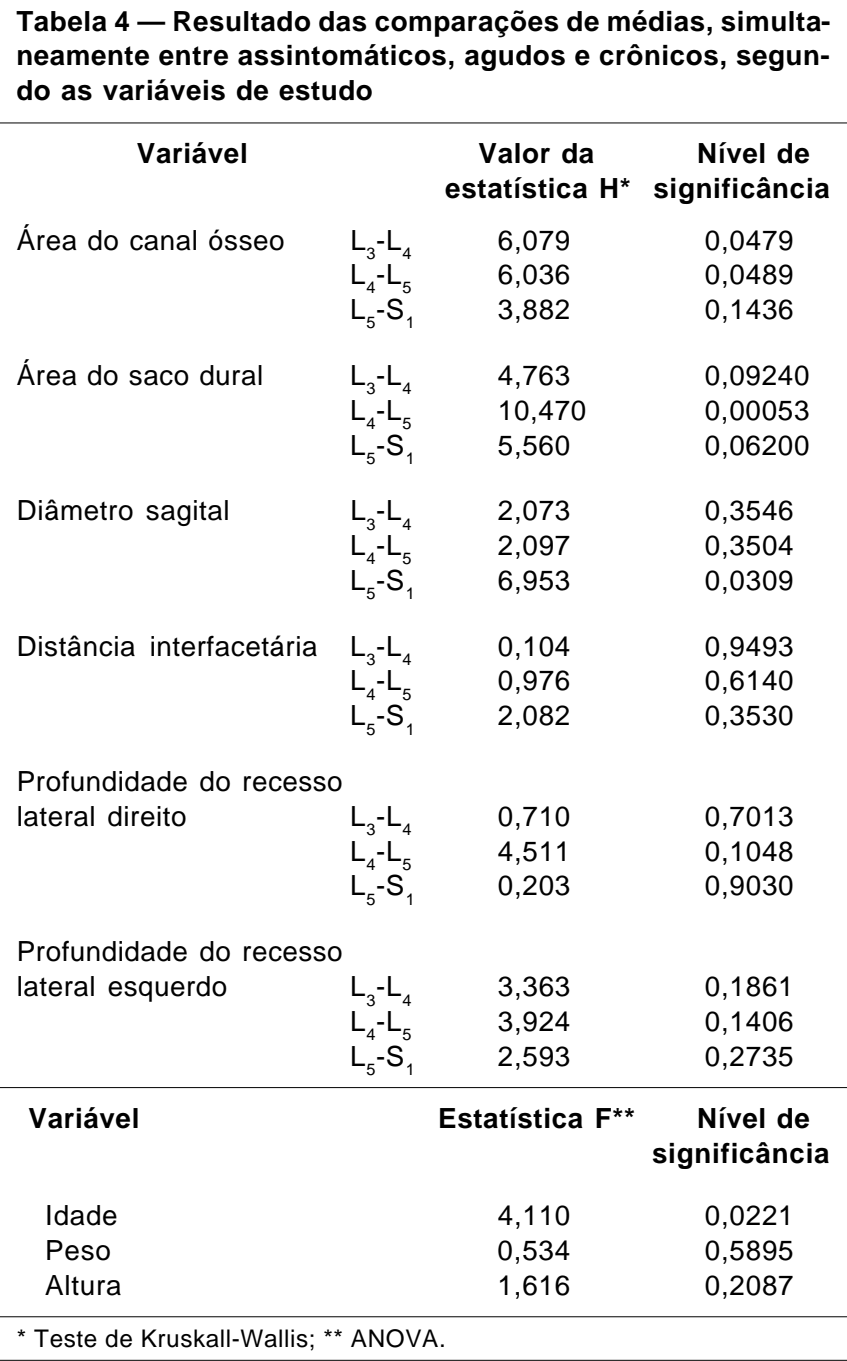

Tabela 5 - Resultado das comparações das médias entre assintomáticos e agudos

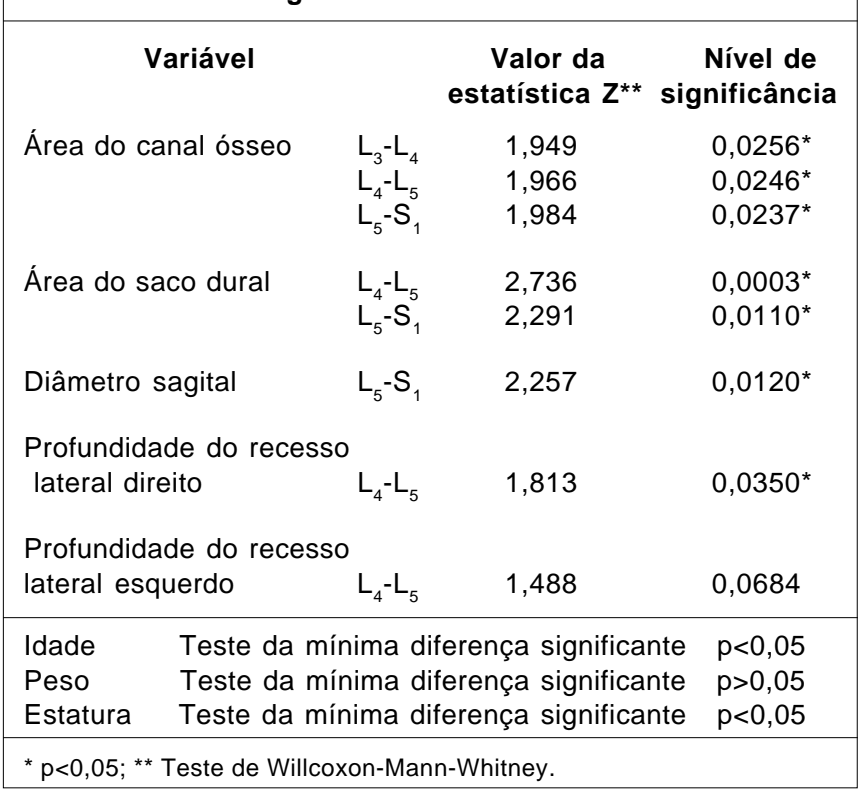

Tabela 6 - Resultados das comparações das médias entre assintomáticos e crônicos

\begin{tabular}{|c|c|c|c|c|}
\hline \multicolumn{3}{|c|}{ Variável } & $\begin{array}{c}\text { Valor da } \\
\text { estatística } Z^{\star *}\end{array}$ & $\begin{array}{c}\text { Nível de } \\
\text { significância }\end{array}$ \\
\hline \multirow{2}{*}{\multicolumn{2}{|c|}{ Área do canal ósseo }} & $\mathrm{L}_{3}-\mathrm{L}_{4}$ & 2,037 & $0,0208^{*}$ \\
\hline & & $\mathrm{L}_{4}-\mathrm{L}_{5}$ & 1,940 & $0,0262^{*}$ \\
\hline \multirow{2}{*}{\multicolumn{2}{|c|}{ Área do saco dural }} & $\mathrm{L}_{4}-\mathrm{L}_{5}$ & 2,350 & $0,0009^{*}$ \\
\hline & & $\mathrm{L}_{5}-\mathrm{S}_{1}$ & 0,398 & $0,0345^{*}$ \\
\hline \multicolumn{5}{|c|}{ Profundidade do recesso } \\
\hline lateral d & reito & $\mathrm{L}_{4}-\mathrm{L}_{5}$ & 1,651 & $0,0494^{*}$ \\
\hline \multicolumn{5}{|c|}{ Profundidade do recesso } \\
\hline lateral e & querdo & $\mathrm{L}_{4}-\mathrm{L}_{5}$ & 1,711 & $0,0435^{*}$ \\
\hline Idade & \multicolumn{3}{|c|}{ Teste da mínima diferença significante } & $p>0,05$ \\
\hline Peso & \multicolumn{3}{|c|}{ Teste da mínima diferença significante } & $p>0,05$ \\
\hline Altura & \multicolumn{3}{|c|}{ Teste da mínima diferença significante } & $p>0,05$ \\
\hline
\end{tabular}

Tabela 7 - Resultado da comparação das médias entre a fase aguda e crônica, segundo as variáveis de estudo

\begin{tabular}{|lccc|}
\hline \multicolumn{1}{|c}{ Variável } & & $\begin{array}{c}\text { Valor da } \\
\text { estatística } Z^{* *}\end{array}$ & $\begin{array}{c}\text { Nível de } \\
\text { significância }\end{array}$ \\
Área do saco dural & $\mathrm{L}_{4}-\mathrm{L}_{5}$ & 1,883 & $0,0299^{*}$ \\
Diâmetro sagital & $\mathrm{L}_{5}-\mathrm{S}_{1}$ & 2,536 & $0,0005^{*}$ \\
\hline $\begin{array}{l}\text { Idade } \\
\text { Peso }\end{array}$ & Teste da mínima diferença significante & $\mathrm{p}>0,05$ \\
Altura & Teste da mínima diferença significante & $\mathrm{p}>0,05$ \\
\hline${ }^{*} \mathrm{p}<0,05 ;{ }^{* *}$ & Teste de Willcoxon-Mann-Whitney. & \\
\hline
\end{tabular}

$\mathrm{Na}$ tabela 5, podemos constatar que as áreas do canal ósseo nos três segmentos ea do saco dural em L4-L 5 e L 5-S1 apresentaram diferenças estatisticamente significantes entre os assintomáticos e sintomáticos em fase aguda. Essa diferença também foi observada em relação ao diâmetro sagital em L 5-S1 eà profundidade do recesso lateral direito em L4-L5. N o que se refere à i dade, peso ealtura, foram constatadas diferenças em relação à idade e estatura, sendo os pacientes agudos mais jovens e de maior estatura.

A tabela 6 indica que os assintomáticos e crônicos são diferentes, sob o ponto de vista estatístico, no que se refere à área do canal ósseo em $L 3-L 4$ e L4-L 5, à área do saco dural em L4-L 5 e L5-S1, e às profundidades dos recessos laterais em L 4-L 5 .

Para determinar diferenças entre pacientes agudos e crônicos, estes tiveram suas variáveis dispostas na tabela 7.

Nessa tabela, observou-se uma diferença estatisticamente significante, em relação à área do saco dural e ao diâmetro sagital em L5-S1. Tam- 
bém os agudos se diferenciaram dos crôni cos quanto à idade.

Sumariando os resultados:

- As variáveis sexo, idade, peso e estatura apresentaram igualdade de variâncias nos testes para homocedasticidade; quando assintomáticos e sintomáticos, foram comparados como um todo. Em outras palavras, esses dois grupos são similares, em termos estatísticos.

- A prevalência de alterações tomográficas e pelo raio $X$ convencional foi estatisticamente semel hante, quando se compararam assintomáticos e sintomáticos.

- Homocedasticidade e simetria foram, ainda, observadas nessa mesma distribuição; quando assintomáticos e sintomáticos, esses agora separados em agudos e crônicos, foram, simultaneamente, comparados entre si. Apenas no que se refere à idade, houve diferença estatisticamente significante, tendo sido constatado, posteriormente, que os agudos são mais jovens que os pacientes crônicos.

- Heterocedasticidade foi observada, no que concerne à distribuição das variáveis referentes aos parâmetros vertebrais.

- Essas variáveis mostraram ter, no grupo assintomático, val ores mai ores, em rel ação ao grupo dos agudos e ao grupo dos crônicos.

- Essa prevalência continuou semelhante quando os últimos foram separados em agudos e crônicos, e o método de estudo foi a tomografia axial computadorizada.

- As áreas de seção transversa do canal ósseo em L 3-L 4 e L4-L5, a área de seção transversa do saco dural em L4-L5, L5-S1 eas profundidades dos recessos laterais em L 4-L5 são menores nos crônicos, quando comparados com os assintomáticos.

- As áreas de seção transversa do canal ósseo em L 3-L 4, L 4-L 5 e L 5-S1; as áreas do saco dural em L4-L5 e L5-S1; o diâmetro sagital em L5-S1 e a profundidade do recesso lateral direito em L4-L5 são, i gual mente, menores nos agudos, quando comparados com os assintomáticos. Esses pacientes tinham maior estatura.

- Os pacientes agudos, quando comparados com os crônicos, mostraram ter, sob o ponto de vista estatístico, diâmetro sagital e saco dural menores em L5-S1. São eles, também, mais jovens.

\section{DISCUSSÃO}

Pela análise dos resultados, e considerando as diferenças estatisticamente significantes encontradas, verificamos que as médias das áreas de seção transversa do canal ósseo em L 3-L 4 e L 4-L5 são maiores nos assintomáticos que nos crôni cos e agudos. Ainda, sob o ponto de vista estatístico, a profundidade dos recessos laterais em L4-L5, nos pacientes crônicos, e o diâmetro sagital em L5-S1, nos pacientes agudos, são diferentes dos assintomáticos, sendo as médias destes maiores que daquel es (tabelas 5 e 6). E $m$ função dessas diferenças estatisticamente significantes, é correto admitir que, nesses segmentos, o canal raquidiano possui dimensões maiores nos assintomáticos.

Nas outras variáveis estudadas, em que não foram constatadas essas diferenças estatisticamente significantes, as médias e medianas, no entanto, tinham valores sistematicamente mais el evados nos assintomáticos do que nos pacientes, sejam esses agudos ou crônicos (tabela 1).

E mbora o valor da estatística dessas poucas médias não tenha alcançado a significância de 5\% proposta, a tendência, observada nos assintomáticos, sinaliza para uma mesma direção, instigadora, apontada por aquelas variáveis estatisticamente significantes, ou seja: os canais raquidianos dos assintomáti cos são mai or es que os dos sintomáticos.

E $m$ relação àquelas diferenças não significativas (ou pouco significativas), encontradas nos nossos resultados - mas consistentes como provas de uma hipótese - , é oportuno trazer o comentário de Chapin ${ }^{76}$, apud Berquó ${ }^{77}$ :

"Nenhuma dessas diferenças são estatisticamente significantes como diferenças singulares. O ponto importante é que el as são tão importantes quanto as diferenças que são amplas e estatisticamente significantes. Nossa opinião é que, inequivocamente, pequenas diferenças na mesma direção podem ser tão importantes quanto grandes diferenças, que sãosignificantes, estatisticamente."

A propósito dessas al egações, e ainda pertinente ao assunto, é lapidar o trocadil ho do cél ebre astrofísico Carl Sagan, apud Day,R.A ${ }^{78}$ : em determinadas circunstâncias, "a ausência de provas não é prova de sua ausência".

Em decorrência das diferenças significantes verificadas (já suficientes como um corpo de prova), e daquel as pouco significantes ou próximas da significância, uma ilação lógica vem à tona: os assintomáticos assim o são porque os seus canais raquidianos, em sendo mais amplos, possivel mente, possuem uma reserva anatomofuncional. Essa possibilidade surge como corolário de dois fatos: 1) as médias da área do canal ósseo, nos assintomáticos, são maiores do que nos crônicos e ainda maiores que nos agudos (tabela 4); 2) a média de idade dos agudos é menor que nos crônicos e assintomáticos. Essas duas verificações, por não terem ocorrido ao acaso (uma vez que for am embasadas em diferenças estatisticamente significan- 
tes), permitem-nos chegar a um terceiro fato, corolário dos dois anteriores: 3) se quadros agudos são predominantes em jovens, se quadros crônicos predominam em idosos, se canais raquidianos são menores em agudos, mai or es em crônicos (em relação a agudos) e tanto uns como outros (agudos e crônicos) menores que em assintomáticos, é de se admitir que essas diferenças são, provavelmente, ocasionadas pela presença de um fator que se distribuiria diferencialmente entre agudos, crônicos e assintomáticos. Esse "fator de segurança"79,80 seria uma reserva anatomofuncional e se constituiria de um espaço preenchido de tecido gorduroso, existente entre a dura-máter e a parte interna do canal ósseo (fig. 1).

A referida reserva seria: menor nos agudos (que, por ser pequena não é capaz de retardar o aparecimento dos sintomas); daí, a maior prevalência de casos agudos nos jovens; um pouco maior nos crônicos (ela é capaz de retardar, mas não impedir, o início dos sintomas até idades mais avançadas); e, ainda maior nos assintomáti cos (que, por ser maior ainda que a dos dois anteriores, é capaz de impedir a produção dos sintomas).

A dedução é lógica e tem fundamento, já que os canais raqui dianos de ambos os grupos apresentam uma semelhante prevalência de alterações tomográficas (tabela 1).

E sse espaço de reserva, acima aludido, inerente aos assintomáticos, por ser maior, funcionaria como uma barreira de proteção às estruturas nervosas diante do processo patológico mecânicodegenerativo, oriundo das estruturas osteoarticulares adjacentes. Contrariamente, nos sintomáticos, essa reserva, por ser menor, faz as referidas estruturas nervosas serem fácil e prontamente alcançáveis pelo citado processo patológico. Uma constatação nesse sentido aponta para o mesmo alvo: a maior prevalência de protrusões discais focais (ou hérnias discais) em L4-L5 e L5-S1, nos pacientes agudos (gráfico 6); as diferenças altamente significativas, entre assintomáticos e agudos, quanto às áreas do saco dural nesses níveis e ao diâmetro sagital L5-S1; e a ocorrência destas protrusões em pessoas de menor idade (tabelas 5 e 7).

I sso significa que o processo compressivo discal, ocorrendo em um canal mais estreito no plano transverso e sagital (chegando ao ponto de reduzir o saco dural ), foi capaz de produzir sintomas aguda e abruptamente, tal a proximidade entre o disco e o saco tecal. Por isso, alguns dos pacientes agudos, com pouca reserva de espaço, apesar de suas hérnias serem pequenas, apresentaram sintomatologia exuberante. Desse modo, o maior ou menor espaço de manobra é o el emento diferenciador ca- paz de separar sintomáticos de assintomáticos. Esses tiveram protrusões focais, e nem por isso apresentaram sintomas (gráfico 4).

Apesar dessa reserva anatomofuncional existir no plano transversal, portanto, de superfície, pressupõe-se que seja, também, uma reserva tridimensional, uma vez que o estojo ósseo raquídeo é um cilindro eixo.

E $m$ razão disso, e levando-se em conta o eixo longitudinal da coluna, pequenas diferenças de área (área do canal ósseo, menos a área do saco dural) podem, às vezes, significar importantes diferenças de volume. E m medidas da ordem de centímetros, variações milimétricas podem ser significativas. Nos outros setores da economia orgânica, a "reserva funci onal" teve sua constatação firmada já há algumas décadas. Nos livros clássicos de Fisiologia e em artigos da literatura, referências são feitas sobre uma "reserva cardíaca", "renal", "pulmonar"45,79-92. Nesses relatos, não observamos nenhuma alusão quanto à sua existência na coluna vertebral. Recentemente, trabal hos foram publicados sobre a sua utilidade em F isiopatol ogia e Terapêuti ca ${ }^{80-93}$. Sua constatação na coluna vertebral indica que esse setor da economia não é uma exceção ao que se observa em outros órgãos e sistemas.

Argumentando: pel o exposto, fica evidente que o tamanho do canal se altera no sentido da diminuição, em razão de desvios dos elementos anatômicos adjacentes que o invadem e, por isso, não há como fugir às evidências de que a fisiopatologia da dor lombar mecânico-degenerativa envolve uma questão de espaço: o confronto de for ças mecânicas em conflito, ou dentro de um espaço maior (e maior "reserva", com pouca ou nenhuma repercussão clínica), ou dentro de um espaço menor (e menor "reserva", com produção de sintomas).

Nossos resultados, ao demonstrarem áreas do canal ósseo menores nos sintomáticos (tanto crônicos, como agudos) queem assintomáti cos (tabelas 5 e 6), confirmam a suspeita levantada por Verbiest $^{94-96}$ e, mais tarde, por Epstein, apud Deburge ${ }^{19}$, apontando para uma possível causa embriológica da reserva anatomofuncional, motivo deste trabaIho: os pacientes com dor lombar apresentam canais raquidianos constitucionalmente mais estreitos, em conseqüência de distúrbios de crescimento do arco neural ${ }^{96}$. Vão ao encontro dessa afirmação as observações clínicas amplamente constatadas na prática médica e nos relatos da literatura ${ }^{71,97-102 .}$

Entrementes, os conflitos disco-vértebro-tecais são mais comuns em L 3-L 4 e L 4-L 5 nos crônicos e L5-S1 nos agudos ${ }^{103}$ (exatamente os mesmos segmentos que, em nossa casuística, revelaram ser 
menos amplos nos pacientes e mais amplos nos assintomáticos).

Daí, pequenas hérnias discais ou discretas alterações degenerativas, nestes locais, causarem intensa sintomatologia em alguns pacientes ${ }^{95}$, ao passo que grandes hérnias, protrusões ou osteófitos causam pouco ou nenhum sintoma em ou$\operatorname{tros}^{63,101}$. I sso foi observado em nossa casuística e já mencionado: hérnias discais, levando a quadros de dor intensa, e hérnias e protrusões volumosas, sem produzir sintomas.

Explica-se: nos primeiros, com canais mais estreitos, por terem uma menor reserva anatomofuncional, tornam-se mais suscetíveis de compressão os elementos nervosos, do que naqueles com canais mais largos (e, portanto, com mai or reserva anatomofuncional).

O aparecimento de sintomas, em pessoas com canais constitucionalmente mais estreitos e, portanto, com menor reserva anatomofuncional, coloca em destaque os fatores mecâni cos na sua produção (compreende-se, então, por que os agudos são mais jovens) $)^{9,47,55,96,102,104,105}$. Como, em última análise, existe uma inter dependência entre esses (fatores mecânicos) e aquela (reserva anatomofunci onal), tais fatores foram intensa e repeti damente invocados até agora.

A diminuição da reserva anatomofuncional, que também propomos seja chamada de "fator de segurança vertebral", ao permitir o atrito entre os elementos ósseos e as raízes nervosas, por um lado, e ao restringir o fluxo sanguíneo já relativamente pobre na medula espinhal ${ }^{9}$ (quando comparados com os nervos periféricos $\left.{ }^{9,106}\right)$, por outro, explica, quando em situação oposta com aumento da "reserva", o porquê da grande freqüência de alterações radiológicas em indivíduos absol utamente assintomáticos, constatada tanto na nossa casuística (gráfi cos 4 e tabela 1) como em vários trabalhos da literatura $26,27,34,37,75,107$. Essas alterações, contidas pel o "fator de segurança vertebral", não atingem os elementos nervosos e vasculares da raque e, por isso, não ocasionam sintomas.

Argumentando em senti do contrário, pressupõese que as pessoas do grupo assintomático, em razão de uma maior "reserva funcional e anatômica", apesar de apresentarem evidentes alter ações patológicas, não mostram sintomas, pois existe um espaço mai or de manobras para as estruturas vasculares e nervosas, que se vão acomodando e se adaptando a estas al terações ao longo do tempo ${ }^{108}$.

Os resultados desta pesquisa, revelando a possível existência de uma reserva funcional e anatômica no canal raquidiano, explicam a razão dos $81,5 \%$ de alterações tomográficas no grupo assin- tomático. O corolário resultante desta alegação confereà propedêutica clínica um papel derelevância no diagnóstico das doenças vertebrai $\mathrm{s}^{10,100,109,110}$ nesta era de avanços tecnol ógi cos. Por isso, é imperativa a valorização da anamnese e do exame físi $\mathrm{CO}^{31,100,109}$, tão ou mais importantes que radiografias e tomografias. Estas, quando não devidamente correlacionadas com dados clínicos ${ }^{111}$, podem ser enganadoras.

Não obstante a tomografia axial computadorizada e mesmo a ressonância nuclear magnética tenham mel horado, de forma surpreendente, a capacidade de visualizar as características morfológicas do osso ${ }^{112}$ e dos tecidos moles da raque $62,113,114,115$, os clínicos e radiologistas tinham dúvi das sobre quais al ter ações são real mente geradoras de sintomas e sinais, ou se as anomalias radiográficas encontradas são pura e simplesmente relacionadas ao processo de envel hecimento ${ }^{116}$. A comprovação, por nós, da existência de uma reserva anatomofuncional no canal raquidiano dá uma explicação para esta justificada dubiedade.

A possibilidade de canais raquidianos estreitos (e, portanto, com nexo causal em relação à tese em discussão) causarem lombalgias e ciatalgias tem uma substancial fundamentação em vários trabaIhos, entre os quais destacamos os seguintes:

Os estudos clássicos de Verbiest ${ }^{94,95}$, citados anteriormente, apontam o estreitamento do canal raquidiano como fonte de dor lombar e ciática. A dedução, propiciada pela situação oposta (canais mais largos protegeriam os indivíduos contra ela), embora tenha sido objeto de conjecturas, não passou (como agora o faz), no entanto, pelo crivo da anál ise estatística de um estudo controlado. A nossa tese, agora, comprova as suspeitas aventadas.

Malcom J ayson ${ }^{6,65}$ argüiu a suspeição de que 0 canal raquidiano normal tem dependência de uma reserva, de tal forma que qualquer estrutura, como prol apso ou osteófito, invadindo esteespaço, desl oca mais do que lesa as raízes nervosas. Não havendo um espaço adicional de sobra, a raiz é prontamente lesada. Esse autor, em recente publicação117, demonstrou que há uma relação estatisticamente significante entre doença degenerativa discal (com proliferação osteofitária e protrusão discal) e compressão de veias epidurais, com dilatação das veias não comprimidas. Além do mais, foi demonstrado, nesses estudos realizados em cadáver, que existe uma relação estatisticamente significante entre a intensidade da protrusão e a evidência de compressão venosa e dilatação.

Considerando a existência de um espaço ${ }^{118,119} a$ separar normalmente o disco e o sistema venoso epidural, élógica a inferência de que, quanto maior 
for esse espaço, menor é a possi bilidade de compressão mecânica das veias epidurais, compressão esta ti da como de importante partici pação na patogênese da dor lombar. E sses estudos, embasados no método estatístico e experimental, consolidam e dão uma sustentação adicional à hipótese testada.

A ocorrência de diferenças significantes, sob o ponto de vista estatístico, tanto na nossa casuística como na literatura ${ }^{120}$, em relação ao diâmetro sagital de L5-S1, entre agudos, crônicos e assintomáticos (tabelas 5 e 7), indica que esse fato não é meramente circunstancial e, sim, uma determinante causal que, possivelmente, deve estar ligado, como previa Verbiest, a uma anomalia de desenvol vimento da coluna vertebral ${ }^{121}$.

E ssa anomal ia seria modulada por um fator, que se distribuía de forma diferencial nos indivíduos, fazendo-os portadores de uma reserva maior ou menor. A procura de explicações não se limitou a suspeitas de Verbiest e Epstein.

Mais recentemente, Weisz e Lee ${ }^{102,122,123}$ e outros $^{118}$, como já assinalamos, deram a sua contribuição. Esses autores, admitindo existir um espaço livre entrea membrana mais externa (paredeóssea e revestimento ligamentar) ea mais interna (dura, gordura epidural e tecido areolar) do canal raquidiano, onde o tecido neural pudesse se movimentar livremente (e que, se pequeno, poderia produzir uma estenose constritiva), determinaram, pela tomografia computadorizada, apenas no plano sagital, os valores desse "espaço livre". Esse espaço, por eles anunciado, foi obtido pela diferença entre os diâmetros sagital do canal ósseo e o sagital do saco dural de 75 pacientes com dor lombar. A medição foi feita em um total de 46, 41 e 23 níveis (respectivamente, L 3, L 4 e L5), "considerados" por eles "normais" (assintomáticos), dos mesmos 75 pacientes. Usaram a mediana para avaliação.

Alguns aspectos do trabalho de Weisz e Lee merecem considerações, por divergirem desta atual pesquisa:

As "medidas" dos segmentos (tidos como "normais"), por eles utilizadas (na realidade eram níveis "normais" de colunas de pacientes, portanto colunas provavelmente doentes), foram de indivíduos com dor lombar, pela impossibilidade, segundo os autores, de se obter indivíduos "normais" (assintomáticos) para esse fim. Em nosso trabal ho, foi usado um grupo-controle, sem qualquer sintoma ou sinal atual e/ ou pregresso.

A demais, não há garantias de que determinados níveis indenes, de colunas doentes, possam ser consi derados "normais" (assintomáticos).

A ausência de alterações radiológicas não garante a inexistência de doença, como a sua presen- ça também não significa enfermidade.

Apenas a diferença entre o diâmetro do canal ósseo e o diâmetro do saco dural pode ser enganadora, porque ela dá idéi a de uma grandeza linear, medindo uma distância entre dois pontos. E m nossa pesquisa, medimos superfície, uma grandeza de área, portanto, fornecedora de uma noção de espaço mais real. Está também estabel ecido que a área do canal ósseo, muito mais que o diâmetro sagital, é o parâmetro mais sensível para avaliar se um canal é estreito ou não ${ }^{42}$. Mas esses fatores mecânicos, rei teradamente al udidos, não estariam associados a outros? Como explicar, então, os episódios de lombalgias (às vezes sem nenhum motivo aparente), alternando-se com períodos de silêncio sintomatológico?

Que fatores precipitariam esses episódios?

É de se supor que não é tão-somente o estresse mecânico direto51,124,125 (apesar de ser o principal) o fator determinante da eclosão de sintomas em canais estreitos. É possível que, em decorrência dele, haja o surgimento, por ação de fatores nocivos ainda não determi nados, de modificações na circulação da cauda eqüina ${ }^{4,126}$. A constatação de claudicação neurogênica intermitente, associada comumente a uma isquemia medular, e observada em $53,3 \%$ dos nossos pacientes (gráfico 3 ), apóia a validade dessa proposta e acena para uma combinação entre fatores mecânicos e vasculares no surgimento das lombalgias e lombociatalgias. A participação do fator vascular isquêmico foi demonstrada por Parke e Watanabe ${ }^{107,126}$, Ooi et al. (apud Mooney ${ }^{8,127}$ ).

Esses estudos experimentais, ao lado das evidências de que a isquemia supramenci onada (presente em canais estenóticos) torna as estruturas nervosas mais sensíveis ao estresse mecâni Co $^{128}$, e a liberação de substância $\mathrm{P}$ (um neurotransmi ssor de destaque na origem da dor lombar) pelas raízes ganglionares dorsais, sujeitas à vibração mecânica ${ }^{85,129}$, é possível conjecturar - para responder àquelas indagações - que a intensidade desses eventos se dilua ou não ocorra em um sistema vascular livre de compressão, cujos componentes transitam livremente no leito vertebral mais amplo e, portanto, com mai or reserva anatomofuncional. Ao contrário, eles aconteceriam quando ela fosse menor.

\section{CONCLUSÕES}

Os indivíduos assintomáticos, portadores de alterações radiológi cas e tomográficas na col una lombar, possuem canais raquidianos mais largos que os sintomáticos, sejam estes agudos ou crônicos. 
Possivelmente, essa diferença seria determinada pela existência de uma reserva anatomofuncional maior nos assintomáticos, menor nos sintomáticos crônicos, e menor ainda nos agudos.

Essa reserva é um espaço livre, preenchido de tecido gorduroso areolar, existente entre a porção mais externa do saco dural e a parte mais interna do canal ósseo que, quanto mais amplo, explicaria a razão de al guns indivíduos não apresentarem dor lombar.

I gualmente, ela explicaria a ausência de sintomas nos indivíduos com alterações radiológi cas na coluna lombar, pois a exi stência desse espaço livre permitiria uma maior acomodação dos elementos nervosos ao processo patológico.

A área do canal ósseo em L 3-L 4, L 4-L 5, a do saco dural em L4-L5, L5-S1, e a profundidade do recessos laterais em L4-L5 nos pacientes crônicos; a área do canal ósseo nos três segmentos, a área do saco dural em L4-L5, L5-S1, e o diâmetro sagital em L 5-S1 nos pacientes agudos, revel aram-se como importantes parâmetros de avaliação.

Quanto menores as suas dimensões, menor a reserva funcional.

Essa reserva resultaria da presença de um "fator de segurança" vertebral que se distribuiria diferencialmente em agudos, crônicos e assintomáticos.

Essa distribuição diferencial da reserva anatomofuncional, ao explicar falsos-positivos nos exames radiológicos (valorizando a propedêutica clínica) e diferenças nas formas de apresentação clínica, consolida a soberania da propedêutica clínica no diagnóstico das Iombal gias e Iombociatalgias mecânico-degenerativas.

Condensação de tese de Professor Titular apresentada à Faculdade de Medicina do Triângulo Mineiro, Uberaba, MG.

\section{SUMMARY}

Anatomicofunctional reserve in the spinal canal, as an interference factor in the psicopathology of low-back pain and mechanicodegenerative lumbo

OвJ ECTIVE. To test the hipothesis of an anatomic and functional reserve in the spinal canal that explains the radiological changes in lumbar spine of asymptomatic individuals, the differences in modalities of clinical presentation and the discre pancies in the prevalence of mechanical and degenerative low back pain and sciaticas.

CASEs AND Methods. Two groups of 27 persons were submitted to computed axial tomography. In one group, the patients presented low back pain and/ or sciaticas. The other formed by matched controlls, showed no signs or symptoms. The bony canal area, dural sac area, interfacets distanceand lateral recesses depth were quantitative assessed.

RESULTS. The asymptomatics ones had larger bony canals than the acute and chronic patients, due to statistical significant differences. The ranges responsible for these differences and whose averages had higher figures in the asymptomatics ones in relation to the chronic patients, were the bony canal areas at L3-L4, L4-L5, the dural sac area and lateral recesses depth at L4-L 5; in relation to acute ones: the bony canal areas at $L 3-L 4$, L4-L5, L5-S1, dural sac area at L4-L5, L5-S1, and sagittal diameter L5-S1 and lateral recesses depth at L4-L5.

Conclusions. The radiological changes in asymptomatic ones, the lack of symptoms in the control group, the presence of chronic and acute features and the discrepancies in the prevalence of Iow back pain wereattributed to the existence of an anatomic and functional reserve. This reserve would be determined by the presence of a "safety factor" which would have differential distribution in asymptomatics, acute and chronic. It would be the el ement able to influence the presence or absence of low back pain in persons with radiological changes [Rev Ass Med Brasil 43(4): 295-310.]

KEY WORDS: Anatomic and functional reserve. Mechanical low back pain. Sciaticas. Spinal canal. Radiological changes in asymptomatic persons.

\section{REFERÊNCIAS BIBLIOGRÁFICAS}

1. Bassam BA. Low back syndromes (The challenge of accurate diagnosis and management). Postgrad Med 1990; 87: 209-15.

2. Chase JA. Spinal stenosis: when arthritis is more than arthritis? Nurs Clin North Am 1991; 26: 53-64.

3. Frymoyer J W. Back pain and sciatica. New Engl J Med 1988; 318: 291-7.

4. Gaskill MF, Lukin R, Wiot J G. Lumbar disc disease and stenosis. Radiol Clin North Am 1991; 29: 753-64.

5. Heliovaara M. Body height, obesity, and risk of herniated lumbar intervertebral disc. Spine 1987; 12: 469.

6. J ayson MIV. Back pain: some new approaches. Med J Aust 1979; 1: 513-6.

7. Mathews J Á. Backache. Br Med. J 1977; 1: 432-4.

8. Mooney V. Where is the lumbar pain coming from? Ann Med 1989; 21: 373-9.

9. Nachemson A. Advances in low-back pain. Clin Orthop Rel Res 1985; 200: 266-78.

10. Riihimaki H. Low-back pain, its origin and risk indicators. Scand J Work Env Health1991; 17: 81-90.

11. Deyo RA, Tsui-Wu YJ. Functional disability due to back pain. A population-based study indicating the importance of socio-economic factors. Arthritis Rheum 1987; 30: 1.247-53. 
12. Augustus A, Stephen LG. Synopsis: workshop on idiopathic low-back pain. Spine 1982 ; 7: 141-9.

13. Deyo RA, Loeser J D, Bigos SJ. Herniated lumbar intervertebral disk. Am Coll Phys 1990; 112: 598-602.

14. Lipson SJ . Low back pain. In Kelley WN, Harris ED, Ruddy $S$, Sledge CB (eds): Textbook of rheumatology, 3th ed. Philadel phia, WB Saunders, 1989; 508-25.

15. Kelsey LJ , Golden LA, Mundt J D. Low back pain prolapsed lumbar intervertebral disc. Rheum Dis Clin North Am 1990; 16: 699-716.

16. Mooney $\mathrm{V}$, Robertson J . The facet syndrome. Clin Orthop Rel Res 1976; 115: 149-56.

17. Nachemson A. Newest knowledge of low back pain. A critical look. Clin Orthop 1992; 279: 8-20.

18. Nixon J . Intervertebral disc mechanics: a review. J R. Soc Med 1986; 79: 100-4.

19. Deburge A. Sciatiques par sténose du canal Iombaire. Presse Med 1984;13: 973-4.

20. Olmarker K, Rydevik B. Pathophysiology of sciatica. Orthop Clin North Am 1991; 22: 223-34.

21. Cecin HA. Alterações observadas na radiologia convencional da coluna lombar em pessoas assintomáticas. Comunicação ao $8^{\circ}$ Curso de Reciclagem em Reumatologia do Triângulo Mineiro, 1990. Uberaba, 1990.

22. Cecin HA. Medições de parâmetros anatômicos da coluna lombar pela tomografia axial computadorizada em assintomáticos e a importância delas no diagnóstico das síndromes lombares. Rev Bras Reumatol 1991; 31: 113-22.

23. Phillips RB, Howe J W, Bustin G. Stress X-rays and the low book pain patient (see coments). J Manip Physiol Ther 1990; 13: 127-33.

24. Boden SD, Davis DO, Dina TS, Patronas NJ, Wiesel SW. Abnormal magnetic-resonance scans of the lumbar spine in asymptomatic subjects. J Bone J oint Surg [Am] 1990; 72-A.

25. Bolender NF, Schonstrom NSR, Spengler DM. Role of computed tomography and myelography in the diagnosis of central spinal stenosis. J BoneJ oint Surg [Br] 1985; 67: 2406.

26. Buirski G. The non-invasive investigation of lumbar disc degeneration in patients with chronic low back pain. Royal Melbourne Hospital, 1989. Australia's Radiol 1989; 33: 2336.

27. Doden SD, Davis OD, Dina TS, Patronas NJ, Wisel SW. Abnormal magnetic-resonance scans of the lumbar spine in asymptomatic subjects. J BoneJ oint Surg.[Am] 1990; 72-A: 403-8.

28. Dorwart RH, Vogler J B, Helms CA. Spinal stenosis. Radiol. Clin. North Am 1983; 21: 301-25.

29. Frymoyer J W, Newberg A, Pope $\mathrm{MH}$ et al. Spine radiographs in patients with low back pain. J. Bone J oint Surg.[Am] 1989; 66-A:1.048-55.

30. Granda J L, Wertheimer TM, Salas J M et al. X-ray changes in the lumbar spine: correlation with age and low back pain. Artrhitis Rheum 1982; 25: S44.

31. Haldeman S, Shouka M, Robboy S. Computed tomography, electrodiagnostic and clinical findings in chronic workers' compensation patients with back and leg pain. Spine 1988; 13: $345-50$

32. Hitselberger WE, Witten RM. Abnormal myelograms in asymptomatic patients. J Neurosurg 1968; 29: 204-6.

33. Lawrence J S, Bremner J M, Bier F. Osteo-arthrosis: prevalence in the population and relationship between symptoms and X-ray changes. Ann Rheum Dis 1966; 25: 1-24.

34. Powell MC, Szpryt P, Wilson M, Symonds EM, Worthington BS. Prevalence of lumbar disc degeneration observed by magnetic resonance in symptomless women. Lancet 1986;
2: $1.366-7$.

35. Slebus FG, Braakman R, Schipper J, Van Dongen KJ, Westendorp-de Serière $M$. Non-correspondig radiological and surgical diagnoses in patients operated for sciatica. Acta Neurochir 1988; 94: 137-43.

36. Svenssoh H, Anderson GBJ . L ow-back pain in 40-to 47 yearold men: work history and work environment factors. Spine 1983; 8: 272.

37. Wiesel SW, Tsourmas N, F effer HL, Citrin CM, Patronas N. A study of computer assisted tomography. The incidence of positive CAT scans in an asymptomatic group of patients. Spine 1984; 9: 549.

38. Witti I, Vestergaard A, Rosenklint A. A comparative analysis of $X$-ray findings of the lumbar spine in patients with and without lumbar pain. Spine 1984; 9: 298-300.

39. Buckle $P$. Epidemiological aspects of back pain within the nursing profession. Int J Nurs Stud 1987; 24: 319.

40. Cady LD, Bischoff DP, O'Connell ER, Thomas PC, Allan J K. Strength and fitness and subsequent back injuries in fire fighters. J Occup Med 1979; 21: 269.

41. Cecin HA, Bichuetti J AN, Minelli C et al. Dor lombar e trabalho pesado: aspectos epidemiológicos. Rev Bras Reumatol 1992; 32: 157-62.

42. Damkot DK, Pope MH, Lord J , Frymoyer J W.The relationship between work history, work environment and low back pain in men. Spine 1984; 9: 395.

43. Kelsey LJ , Githens PN, O'Conner et al. Acute prolapsed lumbar intervertebral disc. An epidemiologic study with special reference to driving automobiles and cigarette smoking. Spine 1984; 9: 608.

44. Kertesz A, Korms R. Low back pain in the workman in Canada. Can Med Assoc J 1976; 115: 901.

45. Lloyd MH, Gauld S, Soutar CA. E pidemiologic study of back pain in miners and office workers. Spine 1986; 11: 136-40.

46. Magora A. Investigation of the relation between low back pain and occupation. Scand J Rehabil Med 1975; 7: 146.

47. Group M, Stanton-Hicks M. Neuroanatomy and pathophysiology of pain related to spinal disorders. Radiol Clin North Am 1991; 29: 665-73.

48. Anderson GBJ. Advances in biomechanics of the spine. ILAR. In: Congress of Rheumatology, 17, 1989, Rio de J aneiro. Proceedings, 1989; 425.

49. Corman LC. Clinical spectrum and treatment of rheumatic syndromes in the elderly. Med Clin North Am 1989; 73: 1.371-81.

50. Han DT. Biomechanical relation of the intervertebral disk, intervertebral foramen and vertebral canal: an experimental study on autopsy specimens. Chung Hua Wai KoTsa Chih 1989; 27: 581-4.

51. Hansson T, Holm S. Clinical implications of vibrationinduced changes in the lumbar spine. Orthop Clin North Am 1991; 22: 247-53.

52. J ayson MIV. General aspects of back pain: an overview. Ilar. In: Congress of Rheumatology, Proceedings, Rio de J aneiro, set. 1989.

53. Lawrence J S. Disc degeneration - its frequency and relationship to symptoms. Ann Rheum Dis 1969; 28: 121-38.

54. Petropoulos B P. Lumbar spinal syndrome. Clin Orthop Rel Res 1989; 246: 70-9.

55. Porter RW. Mechanical disorders of lumbar spine. Ann Med 1989; 21: 361-6.

56. Resnick D, Pate D, Sartoris DJ, Vassiliadis A, Andre M. Stenotic spinal canal models derived from computed tomography data a preliminary investigation. J Comput Assist Tomogr 1987; 11: 51-5.

57. Hirsch $\mathrm{C}$, Ingel mark BE, Miller M. The anatomical basis for 
Iow back pain. Acta Orthop Scand 1963; 33: 1-17.

58. Weinstein PR. The application of anatomy and pathophysiology in the management of lumbar spine disease. Clin Neurosurg 1979:chapter 30: 517-40.

59. Adams MA, Dolan P, Hutton WC, Porter RW. Diurnal changes in spinal mechanics and their clinical significance. J Bone J oint Surg [Br] 1990; 72: 266-70.

60. Hansen $\mathrm{HJ}$. Apud Comparative views on the pathology of disk degeneration in animals. Lab Inv 1959; 8: 1.242-65.

61. Holm S, Maroudas A, Urban J PG, Selstam G, Nachemson A. Nutrition of the intervertebral disc: solute transport and metabolism. Connect Tissue Res 1981; 8: 101-19.

62. Frocrain L, Duvauferrier R, Husson J L et al. Recurrent postoperative sciatica: evaluation with MR imaging and enhanced CT. Radiology 1989; 170: 531-3.

63. Helms CA, Vogler JB. Computed tomography of spinal stenoses and arthroses. Clin Rheum Dis 1983; 9: 417-41.

64. J ackson RP. The facet syndrome. Myth or reality? Clin Orthop 1992; 279: 110-21.

65. Jayson MIV, Million R, Keegan A, Tomlinson I. A fibrinolytic defect in chronic back pain syndromes. Lancet 1984; 24: 1.186-7.

66. Twomey LT, Taylor J R. Age changes in lumbar vertebrae and intervertebral discs. Clin Orthop 1987; 224: 97-103.

67. Yamashita T, Cavanaugh J M, EI-Bohhy AA, GetchelI TV, King Al. Mechanosensitive afferent units in the lumbar facet joint. J Bone J oint Surg [Am] 1990; 72-A: 865-70.

68. Yasuma $\mathrm{T}, \mathrm{K}$ oh $\mathrm{S}$, Okamura $\mathrm{T}, \mathrm{Y}$ amauchi $\mathrm{Y}$. Histological changes in aging lumbar intervertebral discs. J Bone J oint Surg [Am] 1990; 72-A: 220-9.

69. Nachemson A, Andersson GBJ . Classification of low-back pain. Scand J Work Environ. Health 1982; 8: 134-6.

70. Cohen BA, Lanzieri CF, Mendelson DS et al. CT evaluation of the greater sciatic foramen in patients with sciatica. Am J Neuroradiol 1986; 7: 337-42.

71. Resnick D, Niwayama G. Degenerative disease of the spine. In: Diagnosis of bone and joint disorders. 2th ed. Philadelphia, WB Saunders, 1988; 1.480-561.

72. Berquó E, Souza J MS, Gotlieb SLD. Teste de uma variância populacional. In: Bioestatistica. 1a ed. São Paulo, Ed. Pedagógica Universitária, 1981; 247-52.

73. Kreyszig E. Estatística matemática. Mexico (cidade), Limusa Wiley, 1973.

74. Snedecor WG, Cochran WG. Statistical methods. 6th ed. I owa State.University Press, 1967.

75. Buirski G. The investigation of sciatica and low back pain syndromes current trends. Australasian Radiol 1989; 33: 233-6.

76. Chapin FS. Experimental designs in sociological research. New York, Harper, 1947.

77. Berquó ES, Oliveira NCAE, Camargo CPE. A fecundidade em São Paulo. São Paulo: Ed. Brasileira de Ciências, 1977.

78. Day RA. Como escribir y publicar trabajos cientificos. Bol Sanit Panam 1990; 109: 267-88.

79. Silva MR. O coração como bomba. In Aires MM (ed): Fisiologia. 1a ed. Rio de J aneiro, Guanabara Koogan, 1991; 395404.

80. Guyton AC. Insuficiência cardíaca / Ventilação pulmonar. In Guyton: Tratado de fisiologia médica. 8a ed. Rio de J aneiro, Guanabara Koogan, 1992; 215-37 / 352-62.

81. Grant GP, Mansell AL, Granziano J H, Mellins RB. The effect of transfusion on lung capacity, diffusing capacity, and arterial oxygen saturation in patients with thalassemia major. Pediatr Res 1986; 20: 20-3.

82. Keele CA, Eric N. Samson Wright's applied physiology. 10a ed.. London,Oxord University Press, 1961.
83. Kiyohara $\mathrm{H}, \mathrm{K}$ atagiri $\mathrm{H}, \mathrm{N}$ obusawa $\mathrm{S}$ et al. An estimation of functional reserve of the liver in liver cirrhosis using paba test. Nippon Shokakibyo Gakkai Zasshi 1986; 83: 2.028-34.

84. Kumahara $\mathrm{T}$, Muto $\mathrm{Y}$, Moriwaki $\mathrm{H}$ et al. Determination of the integrated CT number of the whole liver in patients with severe hepatitis: as an indicator of the functional reserve of the liver. Gastroenterol J pn 1989; 24: 290-7.

85. Miyagi N. Evaluation of hepatic gluconeogenesis by intravenous $\mathrm{L}$. alanine tolerance test after major hepatectomy. Nippon Shokakibyo Gakkai Zasshi 1989; 86: 2.523-31.

86. Miyata $Y, U$ chida $E$, I shikawa $K$ et al. Hepatic functional reserve and tumor size as prognostic factors in patients with primary liver cancer undergoing non-surgical therapy. Fukuoka I gaku Zasshi 1990; 81: 292-7.

87. Miyoshi S, Minami $Y, K$ awata $S$ et al. Changes in hepatic functional reserve after transcatheter embolization of hepatocellular carcinoma. Assessment by maximal removal rate of indocyanine green. J Hepatol 1988; 6: 332-6.

88. Mizumoto R. Surgery of the liver-past, present and future. Nippon Geka Gakkai Zasshi 1986; 87: 1.499-502.

89. Schutzer KM, Haglund U, Falk A. Cardiopulmonary dysfunction in a feline septic shock model: possible role of leukotrienes. Circ Shock 1989; 29: 13-2.

90. Tanabe $\mathrm{M}$, Tamai $\mathrm{T}$, Mimura $\mathrm{H}$ et al. Clinical values for an index predicting postoperative residual liver function by pre-operative liver-scintigraphy in patients with liver disease. Ann Nucl Med 1989; 3: 25-9.

91. Tepper RS, Morgan WJ , Cota K, Taussig LM. Expiratory flow limitation in infants with bronchopulmonary dysplasia. J Pediatr 1986; 109: 1.040-6.

92. Van Noord J A, Demedts M, Clement J , Cauberghs M, Van de Woestijne KP. Effect of rib cage and abdominal restriction on total respiratory resistance and reactance. J Appl Physiol 1986; 61: 1.736-40.

93. Eisenhauer T, Talartschik J, Hartmann H, Quentin E, Scheler F. Loss of renal functional reserve following kidney transplantation and in patients with advanced disorders of liver function. Klin Wochenschr 1988; 66: 946-52.

94. Verbiest $\mathrm{H}$. A radicular syndrome from developmental narrowing of the lumbar vertebral canal. J BoneJ oint Surg [Br] 1954; 36: 230-7.

95. Verbiest $H$. Further experiences on the pathological influence of a developmental narrowness of the bony lumbar vertebral canal. J Bone J oint Surg [Br] 1955; 37: 576-83.

96. Verbiest $\mathrm{H}$. Developmental stenosis of the bony lumbar vertebral canal. Acta Orthop Belg 1987; 53: 373-87.

97. Arrault I, Benoist J, Rocolle J et al. Valeur diagnostique de la saccoradiculographie et du scanner dans les sténoses lombaires. Diagnostic value of saccoradiculography and scanner in lumbar stenoses. Rev Rhum Mal Osteoartic 1987; 54: 627-31.

98. Dihlmann W. Computertomographie des Iumbalen Diskusprolaps und der vertebralkanalstenose. Z Rheumatol 1984; 43: 153-9.

99. Eisenstein S. Lumbar vertebral canal morphometry for computerised tomography in spinal stenosis. Spine 1983; 8: 187-91.

100. Lipson SJ . Spinal stenosis. Rheum Dis North Am 1988; 14: 613-9.

101. Renier J C, Bontoux I. Le disque intervértébral lombaire. Paris: Encycl Med Chir Appar Locom 1984; 15.840: 10-3.

102. Weisz GM, Lee P. Spinal canal stenosis. Concept of spinal reserve capacity: radiologic, measurements and clinical applications. Clin Orthop 1983;179: 134-40.

103. Martens G, Hoogmartens M. Lumbar spinal stenosis. Acta Orthp Belg 1984; 50: 39-53. 
104. Nachemson A. Towards a better understanding of low-back pain: a review of the mechanics of the lumbar disc. Rheumatol Rehabil 1975; 14: 129-43.

105. Postachini $F$, Massabrio M, Ferro L. Familial lumbar stenosis. J Bone J oint Surg [Am] 1985; 67-A: 321-3.

106. Watanabe R, Parke WW. Vascular and neural pathology of Iumbosacral spinal stenosis. J Neurosurg 1986; 64:64-70.

107. Wilmink J T, Korte J H, Penning L. Dimensions of the spinal canal in individuals symptomatic and non-symptomatic for sciatica: a CT study. Neurol Radiol 1988; 30: 547-50.

108. Spengler DM. Current concepts review. Degenerative stenosis of the lumbar spine. J BoneJ oint Surg [Am] 1987; 69A: 305-8.

109. Shiqing $X, Q$ Quanzhi Z, Dehao F, Anhui H. Significance of the straight-leg-raising test in the diagnosis and clinical evaluation of lower lumbar intervertebral-disc protrusion. J Bone J oint Surg [Am] 1987; 69-A: 517-22.

110. Spratt KF, Lehmann TR, Weinstein J N, Sayre HA. A new approach to the low-back physical examination. Behavioral assessment of mechanical signs. Spine 1990;15: 96-102.

111. Borenstein D. Low back pain: epidemiology, etiology, diagnostic evaluation, and therapy. Curr Opin Rheumatol 1991; 3: 207-17.

112. Stockley I, Getty CJ M, Dixon AK et al. Lumbar lateral canal entrapment: clinical, radiculographic and computed tomographic findings. Clin Radiol 1988; 39: 144-9.

113. Black KA, Mccormick C, Owen ET, Vaughan R. Spinal stenosis: a review of 23 cases, J Rheumatol 1982; 9: 573-8.

114. Modic MT, Masaryk T, Boumphrey F, Goormastic, Bell G. Lumbar herniated disk disease and canal stenosis. Am J Roentgenol 1986; 147: 757-65.

115. Schnebel B, Kingston S, Watkins R, Dillin W. Comparison of $\mathrm{MRI}$ to contrast $\mathrm{CT}$ in the diagnosis of spinal stenosis. Spine 1989;14: 332-7.

116. Bates $D$, Ruggieri $P$. I maging modalities for evaluation of the spine. Radiol Clin North Am 1991; 29: 675-90.

117. J ayson MI. The role of vascular damage and fibrosis in the pathogenesis of nerve root damage. Clin Orthop 1992; 279: 40-8.

118. Chen $\mathrm{KP}, \mathrm{P}$ oon $\mathrm{YY}$, Wong $\mathrm{CH}$. The depth to the epidural space. MaTsui Hsueh Tsa Ch 1989; 27: 353-6.

119. Maillot $C$. The space surrounding the spinal cord. Constitution, organization and relationship with the cerebrospinal fluid. J Radiol 1990; 71: 539-47.

120. Fagerlund MKJ, Thelander U, Friberg S. Size of lumbar disc hernias measured using computed tomography and related to sciatic symptoms. Acta Radiol 1990; 31: 555-8.

121. Bengtsson $B$, Thorson J . Back pain: a study of twins. Acta Genet Med Gemellol 1991; 40: 83-90.

122. Weisz GM. Lumbar canal stenosis an occupational hazard. Int Surg 1986; 71: 199-201.

123. Weisz GM.: Post-traumatic spinal stenosis. Arch Orthop Trauma Surg 1986;106: 57-60.

124. Garfiin SR, Rydevik BL, Brown RA. Compressive neuropathy of spinal nerve roots. A mechanical or biological problem? Spine 1991; 16: 162-6.

125. Kolesnichenko IP, Zinchenko VA, Chesnokov VV. The pathophysiological basis for the treatment of patients with a vertebrogenic pain syndrome localized in the lumbosacral region. Vrach Delo 1991; March(3): 83-4.

126. Parke WW, Watanabe $R$. The intrinsic vasculature of the lumbosacral spinal nerve roots. Spine 1985; 10: 508-15.

127. Ooi $Y$, Mita F, Satoh Y. Pathophysiological study of intermittent claudication in the lumbar canal stenosis. Abstracts of the International Society for the Study of Lumbar Spine. Dallas, International Society for the Study of Lumbar Spine, 1986.

128. Howe J F, Loeser J D, Calvin WH. Mechanosensitivity of dorsal root ganglia and chronically injured axons: a physiological basis for the radicular pain of nerve root compression. Pain 1977; 2: 25-41.

129. Weinstein J. Mechanisms of spinal pain. The dorsal root ganglion and its role as a mediator of low-back pain. Spine 1986; 11: 999-1.001. 